\title{
Pro-Active Asset Entities in Collaborative Networks
}

\author{
Tiago Cardoso and Luis M. Camarinha-Matos
}

Faculty of Science and Technology, Universidade Nova de Lisboa,

Quinta da Torre, Caparica, Portugal

\{tomfc, cam\}@uninova.pt

\begin{abstract}
In a Collaborative Network $(\mathrm{CN})$ scenario it would be desirable that $\mathrm{CN}$ members could be able to start collaboration within their Virtual Organization Breeding Environments or Professional Virtual Communities in a short time-frame, whenever a new Business Opportunity appears.

As a contribution towards such dynamic scenario, this paper proposes the concepts of Assets Entity, Pro-Active Assets Entity and Integrated Market of Pro-Active Assets Entities. The first concept can be used to model attributes and all functional and intelligent content elements a $\mathrm{CN}$ member can provide (its Assets). The second is responsible for the representation and promotion of an Assets Entity. The later offers an adaptation of the market place concept where each Pro-Active Assets Entity registers itself, brokers post their needs and try to find the best providers, through a bidding and negotiation process.
\end{abstract}

Keywords: Collaborative Networks, Assets, Virtual Organization Breeding Environment, Professional Virtual Community, Interoperability.

\section{Introduction}

The area of Collaborative Networks (CNs) has consolidated its conceptual baseline, namely in terms of Reference Modeling, as well as Methods and Tools, identifying distinct perspectives of this scientific discipline, as presented in $[1,2]$. Nevertheless, important critical issues remain regarding the fast creation of such organizations.

Whenever a new Business Opportunity (BO) appears, the $\mathrm{CN}$ members, either companies, other organizations and / or professionals; should be able to start collaboration, within their Virtual Organization Breeding Environment (VBE) or Professional Virtual Community (PVC), in a short time-frame. In the typical, case one of the $\mathrm{CN}$ members - the initiator, or a broker, identifies the needed competencies and takes care of selecting all the needed $\mathrm{CN}$ members in order to create a Virtual Organization (VO) that will satisfy the requirements of the BO.

From the CN members' perspective, when they join this organizational structure, they are already willing to collaborate, adding their value to Virtual Organizations. Two main forms can be identified for this value: 1 - through the provision of services; 2 - through the provision of Intelligent Content. Depending on the economic area of the $\mathrm{CN}$, different examples can be found for these two kinds of Assets: an example of Service Asset can be an assembling process or a consultancy service; an example of 
Intelligent Content Asset can be a business Process Model, a CAD document or even a Multimedia cultural documentary on a given city, for the case of a tourism CN.

Nevertheless, as the $\mathrm{CN}$ evolves, the number of members gets bigger and the chances of an Asset from a specific $\mathrm{CN}$ member to be chosen are reduced. Moreover, current approaches, lack mainly in two perspectives:

1. Passive Entities - Web Services, or content available through WebPages, are passive entities in the sense they stay still waiting for a client initiative instead of behaving in an auto-initiative manner in order to look up for new Business Opportunities and pursue negotiations in order to achieve commitments.

2. No Aggregation - The Assets a $\mathrm{CN}$ member can provide are treated in an independent form - no aggregation is made. If, for example, a Business Opportunity BO1 can be satisfied by 5 distinct assets (A1 ...A5), taking into account that there may be $\mathrm{CN}$ members able to provide more than one of the needed assets, the selection of $\mathrm{CN}$ members to form a VO that will satisfy BO1 can range from 1 up to 5 distinct $\mathrm{CN}$ members. The reduction of the number of $\mathrm{CN}$ members would reduce the needed agreements and potentially improve the work flow but, with current approaches, this reduction remains a coincidence.

Given this context, the following question arises: "Is it possible to create a new conceptual framework able to model the value of $\mathrm{CN}$ members within the organization, composed of new constructs that behave in a social and auto-initiative manner, representing and promoting all the elements that $\mathrm{CN}$ member can provide, in order to improve the tackled Business Opportunities, as well as the business success chances?"

This paper introduces four concepts aligned with this research question:

1. Assets - This concept is the abstraction of distinct kinds of elements a CN member can provide. With this "uniformization", as a base modeling mechanism, the two kinds of assets identified can be treated in the same way whenever a model is created for the formation of a Virtual Organization. For example, the assembling service and some needed CAD document are two assets that can be treated as building blocks in the corresponding VO model - one is a Service and the other is an Intelligent Content.

2. Asset Entities (AE) - The aggregation of all the Assets a $\mathrm{CN}$ member provides, improving the possibilities to reduce the number of $\mathrm{CN}$ members needed for the formation of a VO, as well as the corresponding negotiation effort.

3. Pro-Active Asset Entities (PAE) - Here, auto-initiative is added to the AE concept through the introduction of behavior elements. This concept is inspired in the Multi-Agent Systems (MAS) applied to the AE concept within a CN context. The main objective is to represent an AE and lookup for Business Opportunities where one or more of the represented Assets can be included.

4. Integrated Market (IM) - Finally, in the context of a $\mathrm{CN}$, the concept of an Integrated Market where all the PAEs register themselves and where brokers model and post client's needs - the Business Opportunities (BO). This structure will be the place where PAEs look up for BOs where they can match the Assets they represent. Whenever a match happens, the PAE is responsible for pursuing the needed activities to achieve an agreement, in a bidding-like negotiation process. The scope of an IM can be the one of a VBE (or PVC). 


\section{Contribution to Technological Innovation}

Information and Communication Technology evolution is moving towards autonomy of existing systems and devices, giving them the capacity to observe the surrounding environment, cooperate with neighbors and behave independent of human interaction.

Intelligent Manufacturing, where machines provide their functionality and behave in an auto-initiative basis towards collaborative work, is an example of this reality.

The Internet of Things, where sensorial ability, along with processing and storage, in an auto-initiative social behavior of The Things, is another example.

The contribution of this paper to technological innovation is made through the introduction of four concepts aligned with these trends. These concepts support a development framework aiming the creation of collaborative platforms through an active representation and promotion of $\mathrm{CN}$ member's Assets. This representation provides built-in functionality to carry out tasks like business opportunity search, bidding and negotiation, all in an auto-initiative semi-automated basis.

\section{Related Work}

The proposal of Pro-Active Assets Entity to model Enterprises and Free-lancers assets within Collaborative Networks is mainly inspired in Service Oriented Computing (SOC) and Multi-agent Systems (MAS).

Baseline Concepts and Definitions - Web Services in particular and Service Oriented Computing in general introduced a new abstraction paradigm in software development facilitating inter-enterprises' collaboration. This approach evolved through three main phases that can be identified by their main keywords: Publish - when enterprises became able to "wrap" some functionality and wait for worldwide potential clients to call them; Register / Find - when UDDI registries appeared and web-services became "findable" and finally Compose - when the creation of "Value Added Services" became possible, as a result of the integration of simpler services through mechanisms like workflow or technologies like BPEL4WS. This paradigm presented "a design principle intended for the construction of reliable distributed systems that deliver functionality as a service, with an additional emphasis on loose coupling between interacting services" [4].

Other initiatives, like OSGi, coming from software components research area, provide standard interoperability functionality to compose and change composition of services dynamically. The framework proposed by OSGi Alliance, has already other proposals built on top of it, like Apache FELIX, providing the interoperability means to integrate distinct applications / bundles.

Nevertheless, the Web-Service approach is limited in the sense that services are entities that stay still waiting for a clients' call. Nothing is done by the Web Services themselves to "attract" clients or make "promotions", for example. Furthermore, some authors defend that Web-Services will evolve to big groups, or "Service Parks", "owned" by big players who define the groups' rules [5]. It is not clear yet if this will become the reality, but if it does Small and Medium Enterprises may see their possibility of adoption of this paradigm restricted / subjugated to major players' rules - a potential near-future limitation of this paradigm. However, the organization of SMEs 
as a long term cooperation alliance (such as a VBE or PVC) opens the possibility of creating "service parks" or "service markets" within the alliance.

Recently, in $[6,7]$ the concept of Service Entities (SE) is proposed "as constructs that may help structural and functional CNO modeling". Here, a group of Web-Services can belong to one SE and be enriched with attributes towards better interoperability.

On the other hand, the usage of Multi-agent Systems, as a second inspiration area, represents "a promising approach to both model and implement the complex supporting infrastructures required for virtual enterprises and related emerging organizations" [8]. This work points out the main limitation of this approach as the lack on robustness of current solutions in Wide Area Networks, like the Internet.

Nevertheless, the agents approach presents promising characteristics for this domain. In [9], Wooldridge defines the top 4 Software Agents' properties that constitute important inspiration points for the PAE architecture: autonomy (agents operate without the direct intervention of human or others and have control over their actions and internal state), social ability (agents are able to cooperate with humans or other agents in order to achieve their tasks), reactivity (agents perceive their environment and respond in a timely fashion to changes that occur in it) and pro-activeness (agents do not simply act in response to their environment, they are able to exhibit goal-directed behavior by taking the initiative).

In the case some PAE interaction is needed, typically the case when a single Asset cannot apply to a business opportunity by itself, the production of Value-AddedAssets (VAA) can take place, following workflow interaction patterns between distinct PAEs. Here, inspiration ideas have been retrieved from Multi-agent Systems (MAS), through the existing interaction between distinct Software Agents. [10].

This proposal also extends ideas from the evolution of the Semantic Web, namely the Intelligent Content (I-Content) concept, defined as "information content with explicit semantic description through which machines and people can share meaning and value" [11]. Although the I-Content concept adds semantic to content, it still remains static content. Pro-Active Assets Entity extends the I-Content concept adding pro-activeness and auto-initiative behaviors.

Concerning the little interaction between MAS and SOC research communities; although this is a reality, some initiatives start to connect them. One example is a new trend on the Semantic Web with the introduction of Pro-Activity information in the description of Web Services. Nevertheless, the few initiatives addressing this approach mainly exist in the MAS research area.

In the case of the "Proactivity Layer of the Smart Resource Platform" proposed in [12] or the "Proactive Self-Maintained Resources", described in [13], Semantic Web Languages are used, illustrating this benefit applied to MAS. In both cases, the "agentification" of resources, or smart-devices, is made through this approach. In the first case, the objective was their integration. In the second case, the aim was the definition of an ontology-driven rules' approach for modeling context-sensitive agent behavior.

Web Services and Agent Technologies on Collaborative Networks - In [5], an early proposal was made, defending that "as Web Services become more sophisticated, they effectively will become software agents themselves". The authors defend that WSDL and UDDI are insufficient to support service discovery and composition, mainly because of the lack on semantics. The authors argue that importing ideas from 
Multi-Agent Systems would provide the means to create such sophisticated "Service Agents", needed in the formation of dynamic Virtual Enterprises. This work also points out research directions, like the combination of DAML-S and UDDI approaches.

Another example of work integrating the two paradigms is presented in [14]. There, the authors propose an "embedded Web service architecture (EWSA), inspired in the Software Components' research area, which allows agent-based applications to be integrated into enterprise application environments using Web Services". The main objective of this solution is the reduction of time-to-market of the agent-based applications. Nevertheless, EWSA together with the proposal made in [6] mainly target the formation and evolution life cycle phases of an organization like a CN. Furthermore, these two proposals focus on functional perspectives of enterprises and lack on other kinds of Assets. For example, an enterprise that sells a business process model or an individual that provides touristic multimedia content on European cities, are not covered by any of these approaches.

\section{Research Contribution and Innovation}

Given the assumption of Asset as: anything an enterprise, organization or a freelancer member of a $\mathrm{CN}$ can provide and that can be represented / triggered by a software component; and the assumption of Value Added Asset as: an asset that results from the composition of simpler Assets, or other VAA; the concept of Service Entity, presented in [7], can be extended as Assets Entity - a concept that includes the Assets an entity can provide, as well as a set of Attributes from that entity (an enterprise, another organization or a free-lancer member of a $\mathrm{CN}$ ).

Definition 1 (Assets Entity (AE)) -is a tuple AE=(AT, AS), where:

- $\mathrm{AT}=\left\{\operatorname{attr}_{\mathrm{i}} \mid \mathrm{i} \in \mathrm{N}\right\}-$ the set of Attributes of the corresponding entity.

- $A S=\left\{a_{i} \mid i \in N\right\}-$ the set of Assets the corresponding entity can provide.

Within a context of an increasing number of AEs, new mechanisms to "promote" each AE become useful and needed. The concept of Pro-Active Assets Entity is introduced as a software component responsible for the representation and promotion of an AE, looking for Business Opportunities and behaving in a social manner for the provision of all the Assets of the corresponding Entity. The PAE is defined as follows:

Definition 2 (Pro-Active Assets Entity (PAE)) - is a tuple PAE=(AE, PS, BF), where:

- $\mathrm{AE}$ - The Assets Entity represented and promoted by PAE;

- PS $=\left\{\mathrm{p}_{1}, \mathrm{p}_{2}, \mathrm{p}_{3}\right\}$, a set of 3 Properties defined as follows:

O $\mathrm{p}_{1}$ (Pro-activity) - enabling the PAE to look for Business Opportunities (BOs);

$\circ \mathrm{p}_{2}$ (Negotiation-ability) - enabling the PAE to make bids for discovered BOs in a semi-automated manner, as well as carry out negotiation processes in order to improve previously made bids; 
$\circ \mathrm{p}_{3}$ (Social-ability) - enabling an interaction with other PAEs to support the creation of VOs through IMPs, whenever Asset composition means are needed to apply to a given $\mathrm{BO}$.

- $\mathrm{BF}=\left\{\mathrm{f}_{\mathrm{g}, \mathrm{i}} \mid \mathrm{g} \in\{1\right.$ - General, 2 - I-Content, 3 - Service $\left.\}, \mathrm{i} \in \mathrm{N}\right\}-$ The Built-In Functionality from the PAE, divided into three groups whether the functionality is specific to one of the two kinds of assets (I-Content or Service) or it is common to any kind of asset, as exemplified in Table 1.

Table 1. Example of Pro-Active Assets Entity built-in functionality

\begin{tabular}{l}
\hline $\mathrm{f}_{1,1}-$ Find Correlated PAEs \\
\hline $\mathrm{f}_{1,2}-$ Look up in IMPs for BOs \\
\hline $\mathrm{f}_{1,3}-$ BID on BOs \\
\hline $\mathrm{f}_{1,4}-$ Promote Asset \\
\hline $\mathrm{f}_{1,5}-$ Negotiate Asset for BO \\
$\mathrm{f}_{2,1}-$ Open / Show I-Content \\
$\mathrm{f}_{2,2}-$ Extract Info / Knowledge from I-Content \\
\hline $\mathrm{f}_{3,1}-$ Call / Execute / Call-Back the service Asset \\
\hline $\mathrm{f}_{3,2}-$ Create / Edit VAA.
\end{tabular}

From a provider's perspective, a PAE will work like a promotion helper for its assets, finding Business Opportunities, performing negotiation, and eventually promotions, towards the inclusion of the represented asset in VOs.

Finally, the concept of Integrated Market of PAEs is the place where PAEs register themselves, typically one IMP per VBE / PVC. As an IMP might have a large number of PAEs registered, finding potential compatible Assets within that space is an easier process and improves all the pre-collaboration negotiation effort, as well as guarantees ICT compatibility. These factors improve the possibility of a collaboration to start in a short time-frame window. Furthermore, the IMP performs monitoring and certification functionality on the registered PAEs in order to provide accurate information to support brokers' choices.

Definition 3 (Integrated Market of Pro-Active Assets Entities (IMP)) - is a tuple $\mathrm{IMP}=(\mathrm{PE}, \mathrm{BO}, \mathrm{PM}, \mathrm{CR}, \mathrm{BF})$, where:

$\mathrm{PE}=\left\{\mathrm{pae}_{\mathrm{i}} \mid \mathrm{i} \in \mathrm{N}\right\}-$ the set of registered Pro-Active Assets Entities,

$\mathrm{BO}=\left\{\mathrm{bo}_{\mathrm{i}} \mid \mathrm{i} \in \mathrm{N}\right\}-$ the set of announced business opportunities,

$\mathrm{PM}=\left\{\mathrm{pm}_{\mathrm{i}, \mathrm{j}} \mid\left(\forall \mathrm{pm}_{\mathrm{i}, \mathrm{j}} \exists \mathrm{pae}_{\mathrm{i}} \in \mathrm{PE} \quad \exists \mathrm{bo}_{\mathrm{j}} \in \mathrm{BO}\right)\right\}$ - a set with Performance Measurement (PM) information,

$\mathrm{CR}=\left\{\mathrm{cae}_{\mathrm{i}} \mid\left(\forall\right.\right.$ cae $_{\mathrm{i}} \exists$ pae $\left.\left._{\mathrm{i}} \in \mathrm{PE}\right)\right\}-\mathrm{a}$ set with Certification information on Assets Entities and,

$\mathrm{BF}=\left\{\mathrm{f}_{1}, \mathrm{f}_{2}, \mathrm{f}_{3}, \mathrm{f}_{4}\right\}-4$ Built-In Functionality, where:

$\mathrm{f}_{1}$ (PAE Registration) - enabling distinct PAEs to register themselves, as well as the set of assets from the entity they represent; 
$\mathrm{f}_{2}$ (BO Posting) - enabling brokers to post their needs / BOs;

$\mathrm{f}_{3}$ (Performance Measurement) - providing automated PAE performance measuring mechanisms, as well as enabling brokers to grade their performance, in order to increase the information on every registered PAE. As a result, more accurate data becomes available for future $\mathrm{CN}$ members selection processes.

$\mathrm{f}_{4}$ (Certification) - based on PM info, an IMP can certify some PAE's quality (introducing the notion of conspicuity associated to a Pro-Active Assets Entity).

From the broker's perspective an IMP is a "place" where he can specify some need and wait for distinct bids or proposals. There, instead of asking for the catalogue of some service or content providers, the broker only has to post the need, or Business Opportunity. Furthermore, the IMP concept extends the base ideas from UDDI registry systems, as well as blackboard architectures. The former provides the means needed to give registration functionality for PAEs and the later provides the means needed for Brokers to post their needs - the Business Opportunities.

Process / Motivating Scenario - One of the main contributions of the usage of the proposed concepts come from the changes in the interaction between $\mathrm{CN}$ members and brokers that only start the process specifying a need - the Business Opportunity. After that, the initiative goes to the other side for a specified amount of time where distinct PAEs submit their BIDs. Figure 1 shows a simplified BPMN diagram representing the interaction process between a broker and PAEs through the IMP.

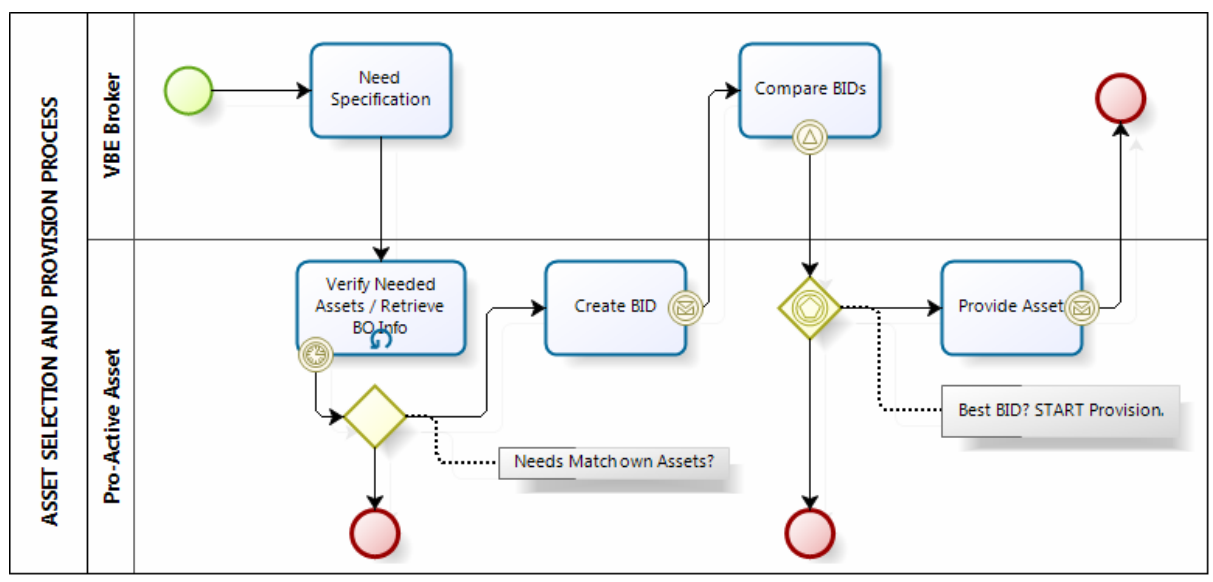

Fig. 1. Broker / PAE Interaction Bidding Process trough IMP

This simplified version of this process is divided into the 5 Tasks $T_{1}$ through $T_{5}$.

$\mathrm{T}_{1}$ (Broker Need Specification) - The VBE / PVC broker divides the BO in simple Asset needs posting them in the IMP. For example "Enterprise A needs a watch production service" or "Person $\mathrm{X}$ wants to go on a tour in the country $\mathrm{Y}$ and is looking for multimedia cultural content concerning city $\mathrm{K}$, as well as accommodation and 
travelling services". In both cases a composed asset can be considered and the broker that gathers this BO forms the VO that will provide a "Value Added Asset".

$\mathrm{T}_{2}$ (BO Analysis) - PAEs gathers BO info, analyze it and decide whether or not to bid. For the first example, PAEs from enterprises with a watch assembling service, for example, analyze the posts while in the second example, PAEs of tourism agents or free-lancers with touristic I-Content from the desired city, perform the same actions.

$\mathrm{T}_{3}$ (Bidding) - Each Pro-Active Assets Entity that finds the posting suitable make a bid and sends it to the broker.

$\mathrm{T}_{4}$ (Best Bid Choice / START) -The broker compares the received bids and chooses the best one. After the choice is made, the selected PAE is notified and the task execution or I-Content transfer can start.

- This choice can go through longer negotiation process between brokers and PAEs, like logistic details definition, for the first example.

$\mathrm{T}_{5}$ (Asset Provision End) - Finally, the process is concluded with the service end or the content provision finishing.

\section{Discussion of Results and Critical View}

Software development history can be divided into two major groups of distinct Development Paradigms (DP): Local-DPs and Distributed-DPs. The evolution from the Local-DPs to the Distributed-DPs' group raised some interoperability challenges concerning the parts to be integrated in the distributed system, namelly:

- the heterogeneity of the environment each part runs on,

- the autonomy of each part and

- the existence of distinct owners of the parts of a system intended to be integrated.

Nevertheless, it is possible to compare some of the major DPs of each group, find similarities on their main constructs and foresee trends.

In the Local-DPs programming, one of the first major DPs was the Functional Programming. There the main building blocks or constructs were the functions or methods. Web-Services, in the Distributed-DPs' group, play a similar role, for the distributed environment. In both cases, the building block is a function or method.

The next major DP, in the Local-DPs, was Object Oriented Programming (OOP) that came in the 60s with Simula. Here, the main building blocks were the Classes of Objects with many methods within a single construct, together with Attributes to store information concerning the modeling elements - the objects. Simula also added some useful features like the Encapsulation, Inheritance and the Polymorphism. Again, a parallel can be established with the Distributed-DPs' group. In 2008 the Service Entities $(\mathrm{SE})$, proposed in $[6,7]$, play a similar role, putting together many web-services with attributes within a single construct - the Service Entities. This time, the keywords in the software development were Functions / Methods and Attributes.

More or less at the same time as the OOP, the Multi-Threaded Programming (MTP) appeared with the corresponding mechanisms for communication and 
synchronization among distinct processes, needed for parallel execution. These two DPs (OOP and MTP) opened the way for the Multi-Agent Systems (MAS). Here, the main building blocks were Software Agents and the new construct was their behavior, other than their autonomy, social ability, reactivity and pro-activeness, as referred in section 3.1. In the Distributed-DPs' group, little before the SEs, technologies like OWL-S (former DAML-S) introduced the semantic constructs needed to "facilitate the automation of Web service tasks including automated Web service discovery, execution, interoperation, composition and execution monitoring".[15]

Establishing a relation between the Local-DPs evolution and the actual reality in the Distributed-DPs, the SE and OWL-S open now a new way for the proposal of solutions that add auto-initiative and behavior constructs to the Functions / Methods and Data Fields / Attributes ones.

Figure 2 synthesizes this comparison exercise on the evolution of Local and Distributed Development Paradigms. The concepts presented in this paper provide a proposal aligned with these trends. The Pro-Active Assets Entity concept represents all the Assets form an enterprise or professional behaving in an auto-initiative manner, looking for business opportunities in Integrated Markets.

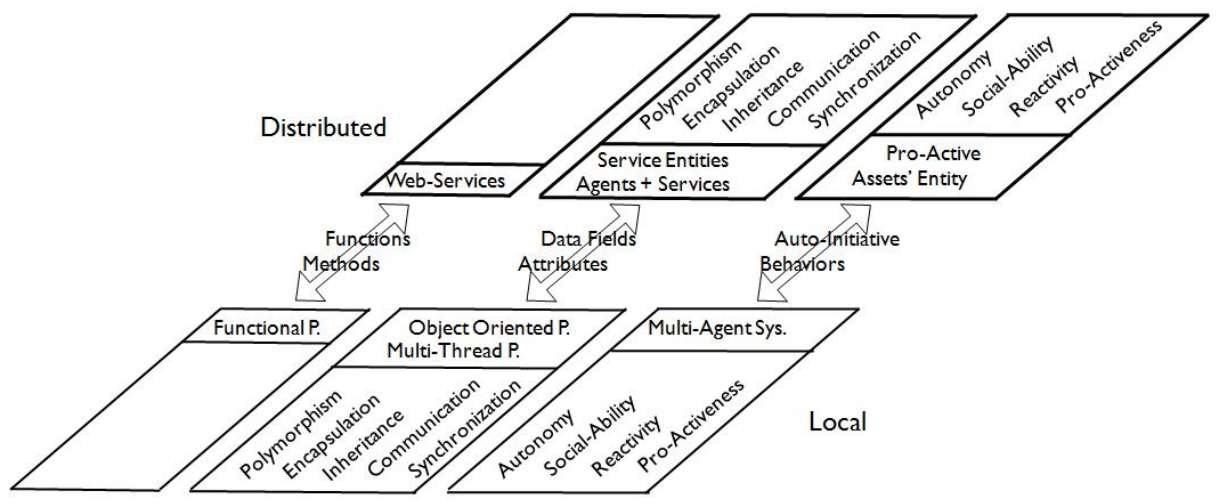

Fig. 2. From Functional Programming to Pro-Active Assets

\section{Conclusions and Future Work}

In this paper a new approach for modeling $\mathrm{CN}$ member's Assets was presented Assets Entities, Pro-Active Assets Entities and Integrated Markets of Pro-Active Assets Entities. The proposal is based on the concept of Asset as something an enterprise, another organization or an individual can provide. This concept extends the functional abilities covered by current approaches, like web-services, to any other kind of Intelligent Content, like a Process Model, for example. On top of this Asset concept, the concept of Assets Entity provides a single construct able to put together all the Attributes and Assets of an Entity (member of a $\mathrm{CN}$ ). The concept of ProActive Assets Entity acts like an envelope that carries the Assets Entity, providing the means to represent it, finding suitable Business Opportunities for it. 
This is done through the introduction of the Integrated Markets. There, the PAEs register themselves and brokers become able to post some needs - the Business Opportunities. The Pro-Active Assets Entities, always looking for suitable Business Opportunities, check for a matching and if the result is positive start a Bidding Process. Finally, the client chooses the BID that best fits the needs.

In section 5, a simple analysis of the evolution of the trends on Development Paradigms shows that this proposal is aligned with the identified directions. In fact, AutoInitiative and Autonomy are two important keywords for systems development.

The ongoing work aimed at the development of a prototype with the base proactiveness and auto-initiative mechanisms. The next phase of the work will target the evaluation of this proposal framework in Collaborative Networks and extend the mode to cover those cases in which no PAE submits a bid to a given Business Opportunity. In this last case, the broker needs to take some other initiatives, namely to convince some PAEs to change their current priorities or alternatively changing $\mathrm{BO}$ requirements.

\section{References}

1. Camarinha-Matos, L.M., Afsarmanesh, H.: Collaborative Networks: Reference Modeling. Springer, New York (2008)

2. Camarinha-Matos, L.M., Hamideh, A., Ollus, M. (eds.): Methods and Tools for Collaborative Networked Organizations. Springer, New York (2008)

3. Brazier, F.: Agents and Service-Oriented Computing for Autonomic Computing - A Research Agenda. IEEE Internet Computing, 82-87 (2009)

4. Srinivasan, L., Traedwell, J.: An overview of service-oriented architecture, web services and grid computing (2005)

5. Petrie, C., Bussler, C.: Service Agents and Virtual Enterprises: A Survey, pp. 68-78 (2003)

6. Franco, R.D., Bas, Á.O., Esteban, F.L.: Modeling extended manufacturing processes with service oriented entities. Service Business 3, 31-50 (2008)

7. Franco, R.D., Bas, Á.O., Burguera, G.P., Varela, R.N.: MANBREE: Supporting Structural and Functional CNO Modeling With Service-Based Entities

8. Camarinha-Matos, L.M.: Multi-Agent Systems in Virtual Enterprises. In: International Conference on AI, Simulation and Planning in High Autonomy Systems (AIS 2002), pp. 27-36. SCS (2002)

9. Wooldridge, M.: Agent-based computing. Interoperable Communication Networks (1998)

10. Rabelo, R.: Advanced Collaborative Business ICT Infrastructures. In: Methods and Tools for Collaborative Networked Organizations, pp. 337-370. Springer, Heidelberg (2008)

11. Intelligent Content (2008)

12. Khriyenko, O.: Proactivity Layer of the Smart Resource in Semantic Web. In: ESWD (2006)

13. Terziyan, V.: SmartResource - Proactive Self-Maintained Resources in Semantic Web: Lessons Learned. IJSH (2007)

14. Ramirez, E.H., Brena, R.F.: Multi-Agent Systems Integration in Enterprise Environments Using Web Services. In: Protogeros, N. (ed.) Agent and Web Service Techn ologies in Virtual Enterprises, pp. 174-189 (2008)

15. DAML Services (2009) 